Journal of Mathematics and Informatics

Vol. 12, 2018, 23-32

ISSN: 2349-0632 (P), 2349-0640 (online)

Published 12 February 2018

www.researchmathsci.org

DOI: http://dx.doi.org/10.22457/jmi.v12a3

Journal of

Mathematics and

Informatics

\title{
Existence of Commutativity in Parikh and Anti-Diagonal Matrices
}

\author{
V. Nithya Vani $^{1}$ and R. Stella Maragatham ${ }^{2}$ \\ Department of Mathematics, Queen Mary's College, Chennai 600004 India \\ nithyavaniv@gmail.com; ${ }^{2}$ rstellamar@yahoo.com \\ ${ }^{I}$ Corresponding author.
}

Received 25 January 2018; accepted 10 February 2018

Abstract. Intensive investigation on various theoretical properties of Parikh matrices has been taken place in the theory of formal languages. This paper deals with the problem of finding properties of words so that their Parikh matrices and Anti-diagonal matrices commute.

Keywords: Parikh mapping, Parikh matrix, Anti-Diagonal matrix, ratio property, Subword.

\section{AMS Mathematics Subject Classification (2010): 68R15}

\section{Introduction}

In this article, the existence of commutative property in Parikh matrix is discussed. We introduce the transpose property of an Anti-diagonal matrix and check the transpose property for the same.

We assume that the reader is familiar with the basics of formal languages. Whenever necessary, [10] may be consulted. The number of occurrences of a word $u$ as a subword in a word $w$, in symbols, $|w|_{u}$. The term subword means that $w$, as a sequence of letters, contains $u$ as a subsequence. This means that there exist words $x_{1}, \ldots, x_{k}$ and $y_{0}, \ldots, y_{k}$ some of them possibly empty, such that $u=x_{1}, \ldots, x_{k}$ and $w=y_{0} x_{1} y_{1} \ldots x_{k} y_{k}$. Subwords in this sense are often called scattered subwords.

The Parikh matrix of a word which has been recently introduced [6] as an extension of the notion of Parikh vector gives more numerical information about the word in terms of certain subwords (also called scattered subwords [9]) than given by the Parikh vector. Since the introduction of this notion of Parikh matrix of a word, there has been an intensive investigation on various theoretical properties of Parikh matrices (See for example $[2,3,4,5,6,7])$.

The Parikh matrix mapping introduced in [2] is a morphism $\psi_{M_{k}}: \Sigma^{*} \rightarrow M_{k+1}$ where $M_{k+1}$ is a collection of $(k+1)$-dimensional upper-triangular matrices with nonnegative integral entries and unit diagonal. The classical Parikh vector $\psi(w)$ appears in the image matrix as the second diagonal. 


\section{NithyaVani and R. Stella Maragatham}

An Anti-diagonal matrix introduced in [8] is a morphism $\delta_{M_{k}}: \Sigma^{*} \rightarrow M_{k+1}$ where $M_{k+1}$ is a collection of $(k+1)$-dimensional right lower-triangular matrices with nonnegative integral entries and right diagonal is unit diagonal. The classical Antidiagonal vector $\delta(w)$ appears in the image matrix as the right lower second diagonal.

To get more information about a word, one has to focus the attention to subwords and factors. In this article, these notions are understood as follows.

\section{Preliminaries}

In this section, we recall subwords, Parikh matrices and anti-diagonal matrices of a word.

\section{Subwords}

Let $\Sigma$ be an alphabet. The set of all words over $\Sigma$ is denoted $\Sigma^{*}$ and the empty word is $\lambda$. If $w \in \Sigma^{*}$, then $|w|$ denotes the length of $w$.

Subword is denoted by $|w|_{u}$ the number of occurrences of word $u$ as a subword in $w$, that is the number of mappings that can be defined with respect to the above definition.

For instance, $|a b b a|_{b a}=2$ and $|a a b b c|_{a b c}=4$.

\section{Parikh matrices}

The notion of Parikh matrix was introduced in [2]. All definitions and results presented in this subsection can be found in $[1,2,3]$. We recall the definition of a Parikh matrix mapping introduced and studied in [2]

Definition 2.1. Let $\Sigma=\left\{a_{1}<a_{2}<\cdots<a_{k}\right\}$ be an ordered alphabet. The Parikh matrix mapping, denoted $\psi_{\Sigma, \mathrm{k}}$, is the monoid morphism: $\psi_{\Sigma, \mathrm{k}}:\left(\Sigma^{*}, \cdot, \lambda\right) \rightarrow\left(M_{k+1}\right.$, , $\left.I_{k+1}\right)$, defined by the condition: if $\psi_{\Sigma, \mathrm{k}}\left(a_{q}\right)=\left(m_{i, j}\right)_{1 \leq i, j \leq(k+1)}$, then for each $1 \leq i \leq$ $(k+1)$,

$m_{i, i}=1, m_{q, q+1}=1$, and all other elements of the matrix $\psi_{\Sigma, \mathrm{k}}\left(a_{q}\right)$ are 0 .

For the ordered alphabet $\Sigma=\left\{a_{1}<a_{2}<\cdots<a_{k}\right\}$, we denote by $a_{i, j}$ the word $a_{i} a_{i+1} \ldots a_{j}$, where $1 \leq i \leq j \leq k$.

The following theorem characterizes the entries of the Parikh matrix:

Theorem 2.1. Let $\Sigma=\left\{a_{1}<a_{2}<\cdots<a_{k}\right\}$ be an ordered alphabet and $w \in \Sigma^{*}$. The matrix $\psi_{\Sigma}(w)=\left(m_{i, j}\right)_{1 \leq i, j \leq(k+1)}$, has the following properties:

- $m_{i, j}=0$, for all $1 \leq j<i \leq(k+1)$,

- $m_{i, i}=1$, for all $1 \leq i \leq(k+1)$,

- $m_{i, j+1}=|w|_{a_{i, j}}$, for all $1 \leq i \leq j \leq k$.

Let $M=\left(m_{i, j}\right)_{1 \leq i, j \leq k}$ be a triangular matrix. The alternate matrix of $M$, denoted by $\bar{M}$, is the matrix $\bar{M}=\left(m_{i, j}^{\prime}\right)_{1 \leq i, j \leq k}$, where $m_{i, j}^{\prime}=(-1)^{i+j}\left(M_{i, j}\right)$ for all $1 \leq i, j \leq k$. The reverse of $M$, denoted by $M^{(r e v)}$, is the matrix $M^{(r e v)}=\left(m_{i, j}^{\prime \prime}\right)_{1 \leq i, j \leq k}$, where $m_{i, j}^{\prime \prime}=$ $m_{k+1-j, k+1-i}$, for all $1 \leq i<j \leq k$. (The entries below the main diagonal are the same in $M$ and $M^{(r e v)}$.) 
Existence of Commutativity in Parikh and Anti-Diagonal Matrices

Definition 2.2. [9] Two words $w_{1}, w_{2}$ over $\Sigma=\{a<b<c\}$ are said to satisfy the ratio property, written $w_{1} \sim_{r} w_{2}$, if $\begin{aligned} \psi_{3}\left(w_{1}\right) & =\left(\begin{array}{cccc}1 & p_{1} & p_{1,2} & p_{1,3} \\ 0 & 1 & p_{2} & p_{2,3} \\ 0 & 0 & 1 & p_{3} \\ 0 & 0 & 0 & 1\end{array}\right) \text { and } \\ \psi_{3}\left(w_{2}\right) & =\left(\begin{array}{cccc}1 & q_{1} & q_{1,2} & q_{1,3} \\ 0 & 1 & q_{2} & q_{2,3} \\ 0 & 0 & 1 & q_{3} \\ 0 & 0 & 0 & 1\end{array}\right) \text { satisfy the condition: }\end{aligned}$

$p_{i}=s . q_{i}(i=1,2,3), p_{i, i+1}=s \cdot q_{i, i+1},(i=1,2)$, where $s$ is a constant.

Now we recall the new notion of Anti-diagonal matrix of a word.

\section{Anti-diagonal matrix}

The notion of anti-diagonal matrix was introduced in [8]. All definitions and results presented in this subsection can be found in [8]. The definition of the anti-diagonal matrix mapping presented below uses a special type of matrix product, called Antidiagonal matrix product.

The notion of an anti-diagonal matrix of a word consider ternary alphabets only. For integers $a_{i}$ and $b_{i}(1 \leq i \leq 3)$, we define the product

$$
\begin{aligned}
& \left(\begin{array}{l}
a_{1} \\
a_{2} \\
a_{3}
\end{array}\right)\left(\begin{array}{lll}
b_{1} & b_{2} & b_{3}
\end{array}\right)=a_{3} b_{1}+a_{2} b_{2}+a_{1} b_{3} \text {. For two matrices } \\
& M_{1}=\left(\begin{array}{lll}
a_{1} & a_{4} & a_{7} \\
a_{2} & a_{5} & a_{8} \\
a_{3} & a_{6} & a_{9}
\end{array}\right), M_{2}=\left(\begin{array}{ccc}
b_{1} & b_{2} & b_{3} \\
b_{4} & b_{5} & b_{6} \\
b_{7} & b_{8} & b_{9}
\end{array}\right) \\
& \text { we define } M_{1} * M_{2}=\left(\begin{array}{lll}
c_{1} & c_{4} & c_{7} \\
c_{2} & c_{5} & c_{8} \\
c_{3} & c_{6} & c_{9}
\end{array}\right) \text { where } c_{i}=\left(\begin{array}{c}
a_{i} \\
a_{i+1} \\
a_{i+2}
\end{array}\right)\left(\begin{array}{lll}
b_{i} & b_{i+1} & b_{i+2}
\end{array}\right) \text { for } i=1,4,7
\end{aligned}
$$

where the product defining $c_{i}$ is as defined above.

Let $\Sigma=\{a, b, c\}$ with $a<b<c$. Then we define

$$
M_{4}(a)=\left(\begin{array}{llll}
0 & 0 & 0 & 1 \\
0 & 0 & 1 & 1 \\
0 & 1 & 0 & 0 \\
1 & 0 & 0 & 0
\end{array}\right),
$$


V. NithyaVani and R. Stella Maragatham

$$
\begin{aligned}
M_{4}(b) & =\left(\begin{array}{cccc}
0 & 0 & 0 & 1 \\
0 & 0 & 1 & 0 \\
0 & 1 & 1 & 0 \\
1 & 0 & 0 & 0
\end{array}\right) \text { and } \\
M_{4}(c) & =\left(\begin{array}{llll}
0 & 0 & 0 & 1 \\
0 & 0 & 1 & 0 \\
0 & 1 & 0 & 0 \\
1 & 1 & 0 & 0
\end{array}\right)
\end{aligned}
$$

This can be formulated as an anti-diagonal matrix $\delta_{3}$ for a ternary word $w$ as

$$
\delta_{3}(w)=\left(\begin{array}{rrrc}
0 & 0 & 0 & 1 \\
0 & 0 & 1 & |w|_{a} \\
0 & 1 & |w|_{b} & |w|_{a b} \\
1 & |w|_{c} & |w|_{b c} & |w|_{a b c}
\end{array}\right)
$$

where $|w|_{a}$ is the number of $a$ in $w,|w|_{b}$ is the number of $b$ in $w,|w|_{c}$ is the number of $c$ in $w,|w|_{a b}$ is the number of $a b$ in $w,|w|_{b c}$ is the number of $b c$ in $w$ and $|w|_{a b c}$ is the number of $a b c$ in $w$.

For example,

$$
\begin{aligned}
& M_{4}(a b c)=\delta_{3}(a) * \delta_{3}(b) * \delta_{3}(c) \\
& \quad=\left(\begin{array}{lllll}
0 & 0 & 0 & 1 \\
0 & 0 & 1 & 1 \\
0 & 1 & 0 & 0 \\
1 & 0 & 0 & 0
\end{array}\right) *\left(\begin{array}{cccc}
0 & 0 & 0 & 1 \\
0 & 0 & 1 & 0 \\
0 & 1 & 1 & 0 \\
1 & 0 & 0 & 0
\end{array}\right) *\left(\begin{array}{llll}
0 & 0 & 0 & 1 \\
0 & 0 & 1 & 0 \\
0 & 1 & 0 & 0 \\
1 & 1 & 0 & 0
\end{array}\right)
\end{aligned}
$$

Using anti-diagonal matrix product, we get

$$
=\left(\begin{array}{llll}
0 & 0 & 0 & 1 \\
0 & 0 & 1 & 1 \\
0 & 1 & 1 & 1 \\
1 & 1 & 1 & 1
\end{array}\right)
$$

\section{Ratio property in an anti-diagonal matrix}

Definition 2.3. Two words $w_{1}, w_{2}$ over $\Sigma=\{a<b<c\}$ are said to satisfy the ratio property, written as

$$
w_{1} \sim_{r} w_{2} \text {, if } \delta_{3}\left(w_{1}\right)=\left(\begin{array}{cccc}
0 & 0 & 0 & 1 \\
0 & 0 & 1 & p_{1} \\
0 & 1 & p_{2} & p_{1,2} \\
1 & p_{3} & p_{2,3} & p_{1,3}
\end{array}\right) \text { and } \delta_{3}\left(w_{2}\right)=\left(\begin{array}{cccc}
0 & 0 & 0 & 1 \\
0 & 0 & 1 & q_{1} \\
0 & 1 & q_{2} & q_{1,2} \\
1 & q_{3} & q_{2,3} & q_{1,3}
\end{array}\right)
$$

satisfy the condition $p_{i}=s \cdot q_{i}(i=1,2,3), p_{i, i+1}=s \cdot q_{i, i+1},(i=1,2)$, where $\mathrm{s}$ is a constant.

\section{Existence of commutativity in Parikh matrices}

In this section, we examine commutative property in Parikh matrix of a word.

Lemma 3.1. Let $\Sigma=\{a, b\}$ with $a<b$ and $w_{1}, w_{2} \in \Sigma$. If $w_{2}$ was got by replacing the word $\boldsymbol{a} \boldsymbol{b}$ to $\boldsymbol{b} \boldsymbol{a}$ (or) $\boldsymbol{b} \boldsymbol{a}$ to $\boldsymbol{a} \boldsymbol{b}$ in any one place of $w_{1}$, then $\psi_{2}\left(w_{1} w_{2}\right)=\psi_{2}\left(w_{2} w_{1}\right)$. 
Existence of Commutativity in Parikh and Anti-Diagonal Matrices

Proof: Let $\Sigma=\{a, b\}$ with $a<b$ and $w_{1}, w_{2} \in \Sigma$. Let $\psi_{2}\left(w_{1}\right)=\left(\begin{array}{ccc}1 & p & r \\ 0 & 1 & q \\ 0 & 0 & 1\end{array}\right)$.

In $\psi_{2}\left(w_{1}\right),\left|w_{1}\right|_{a}=p,\left|w_{1}\right|_{b}=q$ and $\left|w_{1}\right|_{a b}=\mathrm{r}$.

Given that $w_{2}$ is replacing the word $\boldsymbol{a} \boldsymbol{b}$ to $\boldsymbol{b} \boldsymbol{a}$ (or) $\boldsymbol{b} \boldsymbol{a}$ to $\boldsymbol{a} \boldsymbol{b}$ in any one place of $w_{1}$.

We get $\psi_{2}\left(w_{2}\right)=\left(\begin{array}{ccc}1 & p & s \\ 0 & 1 & q \\ 0 & 0 & 1\end{array}\right)$

In $\psi_{2}\left(w_{2}\right),\left|w_{2}\right|_{a}=p,\left|w_{2}\right|_{b}=q$ and $\left|w_{2}\right|_{a b}=$ s. $\psi_{2}\left(w_{1} w_{2}\right)=\psi_{2}\left(w_{1}\right) \psi_{2}\left(w_{2}\right)$

$$
=\left(\begin{array}{lll}
1 & p & r \\
0 & 1 & q \\
0 & 0 & 1
\end{array}\right)\left(\begin{array}{lll}
1 & p & s \\
0 & 1 & q \\
0 & 0 & 1
\end{array}\right)
$$

Using matrix multiplication, we get

$$
\begin{aligned}
& =\left(\begin{array}{ccc}
1 & 2 p & s+p q+r \\
0 & 1 & 2 q \\
0 & 0 & 1
\end{array}\right) \\
& \psi_{2}\left(w_{2} w_{1}\right)=\psi_{2}\left(w_{2}\right) \psi_{2}\left(w_{1}\right) \\
& =\left(\begin{array}{ccc}
1 & p & s \\
0 & 1 & q \\
0 & 0 & 1
\end{array}\right)\left(\begin{array}{ccc}
1 & p & r \\
0 & 1 & q \\
0 & 0 & 1
\end{array}\right)=\left(\begin{array}{ccc}
1 & 2 p & r+p q+s \\
0 & 1 & 2 q \\
0 & 0 & 1
\end{array}\right)
\end{aligned}
$$

Hence, $\psi_{2}\left(w_{1} w_{2}\right)=\psi_{2}\left(w_{2} w_{1}\right)$.

Now we illustrate the above result.

Remark 3.1. Note that we consider binary alphabets in the above results in Parikh matrix of words. But this result is not possible for three letter words in Parikh matrix.

Lemma 3.2. Let $\Sigma_{3}=\{a, b, c\}$ with $a<b<c$. Let $w_{1}, w_{2}, w_{3}$ and $w_{4} \in \Sigma_{3}^{*}$ such that $w_{1}, w_{2}, w_{3}$ and $w_{4}$ satisfy the ratio property $w_{1} \sim_{r} w_{2}, w_{3} \sim_{r} w_{1}$ and $w_{4} \sim r w_{2}$. Then $w_{1} \sim_{r} w_{4}, w_{3} \sim_{r} w_{4}$ and $w_{3} \sim_{r} w_{2}$.

Proof: Let $w_{1}, w_{2}, w_{3}$ and $w_{4}$ over $\Sigma_{3}=\{a<b<c\}$ are said to satisfy the ratio property, written $w_{1} \sim_{r} w_{2}, w_{3} \sim_{r} w_{1}$ and $w_{4} \sim_{r} w_{2}$.

If $\begin{aligned} \psi_{3}\left(w_{1}\right) & =\left(\begin{array}{cccc}1 & p_{1} & p_{1,2} & p_{1,3} \\ 0 & 1 & p_{2} & p_{2,3} \\ 0 & 0 & 1 & p_{3} \\ 0 & 0 & 0 & 1\end{array}\right), \\ \psi_{3}\left(w_{2}\right) & =\left(\begin{array}{cccc}1 & q_{1} & q_{1,2} & q_{1,3} \\ 0 & 1 & q_{2} & q_{2,3} \\ 0 & 0 & 1 & q_{3} \\ 0 & 0 & 0 & 1\end{array}\right),\end{aligned}$ 
V. NithyaVani and R. Stella Maragatham

$\begin{aligned} \psi_{3}\left(w_{3}\right) & =\left(\begin{array}{cccc}1 & u_{1} & u_{1,2} & u_{1,3} \\ 0 & 1 & u_{2} & u_{2,3} \\ 0 & 0 & 1 & u_{3} \\ 0 & 0 & 0 & 1\end{array}\right) \text { and } \\ \psi_{3}\left(w_{4}\right) & =\left(\begin{array}{cccc}1 & v_{1} & v_{1,2} & v_{1,3} \\ 0 & 1 & v_{2} & v_{2,3} \\ 0 & 0 & 1 & v_{3} \\ 0 & 0 & 0 & 1\end{array}\right)\end{aligned}$

If $w_{1} \sim r w_{2}$, we have $\frac{p_{1}}{q_{1}}=\frac{p_{2}}{q_{2}}=\frac{p_{3}}{q_{3}}=\frac{p_{1,2}}{q_{1,2}}=\frac{p_{2,3}}{q_{2,3}}=s$

If $w_{3} \sim{ }_{r} w_{1}$, we have $\frac{u_{1}}{p_{1}}=\frac{u_{2}}{p_{2}}=\frac{u_{3}}{p_{3}}=\frac{u_{1,2}}{p_{1,2}}=\frac{u_{2,3}}{p_{2,3}}=t$

If $w_{4} \sim r w_{2}$, we have $\frac{v_{1}}{q_{1}}=\frac{v_{2}}{q_{2}}=\frac{v_{3}}{q_{3}}=\frac{v_{1,2}}{q_{1,2}}=\frac{v_{2,3}}{q_{2,3}}=l$

By eqn., (1)

$\frac{p_{1}}{q_{1}}=\mathrm{s}$

Using eqn., (3), we get

$\begin{array}{ll}\frac{p_{1}}{\frac{v_{1}}{l}}=s=>\frac{l p_{1}}{v_{1}}=s=>\frac{p_{1}}{v_{1}}=\frac{s}{l}=k & {\left[\because \frac{s}{l}=k\right]} \\ & \frac{p_{1}}{v_{1}}=k\end{array}$

Likewise, $\frac{p_{2}}{v_{2}}=\frac{p_{3}}{v_{3}}=\frac{p_{1,2}}{v_{1,2}}=\frac{p_{2,3}}{v_{2,3}}=k$, which means $w_{1} \sim_{r} w_{4}$

By eqn., (2)

Using eqn., (1), we get

$$
\frac{u_{1}}{p_{1}}=t
$$

Using eqn., (3), we get

$$
\frac{u_{1}}{s q_{1}}=t=>\frac{u_{1}}{q_{1}}=s t
$$

$$
\begin{gathered}
\frac{u_{1}}{\frac{v_{1}}{l}}=s t=>\frac{l u_{1}}{v_{1}}=s t=>\frac{u_{1}}{v_{1}}=\frac{s t}{l}=m \quad\left[\because \frac{s t}{l}=k\right] \\
\frac{u_{1}}{v_{1}}=m
\end{gathered}
$$

Likewise, $\frac{u_{2}}{v_{2}}=\frac{u_{3}}{v_{3}}=\frac{u_{1,2}}{v_{1,2}}=\frac{u_{2,3}}{v_{2,3}}=m$, which means $w_{3} \sim_{r} w_{4}$

By eqn., (2)

Using eqn., (1), we get

$$
\frac{u_{1}}{p_{1}}=t
$$

Likewise, $\frac{u_{2}}{q_{2}}=\frac{u_{3}}{q_{3}}=\frac{u_{1,2}}{q_{1,2}}=\frac{u_{2,3}}{q_{2,3}}=n$, which means $w_{3} \sim_{r} w_{2}$

Hence, $w_{1} \sim_{r} w_{4}, w_{3} \sim_{r} w_{4}$ and $w_{3} \sim_{r} w_{2}$. 
Existence of Commutativity in Parikh and Anti-Diagonal Matrices

Theorem 3.1. Let $\Sigma_{3}=\{a, b, c\}$ with $a<b<c$. Let $w_{1}, w_{2}, w_{3}$ and $w_{4} \in \Sigma_{3}^{*}$ such that $w_{1}, w_{2}, w_{3}$ and $w_{4}$ satisfy the ratio property $w_{1} \sim_{r} w_{2}, w_{3} \sim_{r} w_{1}$ and $w_{4} \sim_{r} w_{2}$.

Then (i) $\psi_{3}\left(w_{1} w_{4}\right)=\psi_{3}\left(w_{4} w_{1}\right)$,

(ii) $\psi_{3}\left(w_{3} w_{4}\right)=\psi_{3}\left(w_{4} w_{3}\right)$ and

(iii) $\psi_{3}\left(w_{2} w_{3}\right)=\psi_{3}\left(w_{3} w_{2}\right)$

Proof: Let $\Sigma_{3}=\{a, b, c\}$ with $a<b<c$. Let $w_{1}, w_{2}, w_{3}, w_{4} \in \Sigma_{3}^{*}$ and $w_{1}, w_{2}, w_{3}$ and $w_{4}$ satisfy the ratio property such that $w_{1} \sim_{r} w_{2}, w_{3} \sim_{r} w_{1}$ and $w_{4} \sim_{r} w_{2}$.

From Lemma 3.2, since $w_{1} \sim_{r} w_{4}, w_{3} \sim_{r} w_{4}$ and $w_{3} \sim_{r} w_{2}$.

If $w_{1} \sim r w_{4}$, From i) of Lemma 1 in [9]

Hence $\psi_{3}\left(w_{1} w_{4}\right)=\psi_{3}\left(w_{4} w_{1}\right)$.

Likewise, ii) and iii) are proved.

\section{Existence of commutativity in an anti-diagonal matrices}

In this section we examine transpose property in an Anti-diagonal matrix of a word.

Proposition 4.1. The transpose of an Anti-diagonal matrix is also an anti-diagonal matrix.

Remark 4.1. Here the Anti-diagonal matrix differs from Parikh matrix as its transpose is not at all a Parikh matrix.

Corollary 4.1. Transpose of an Anti-diagonal matrix is not the same as the reverse matrix as given in Parikh matrices [2].

Example 4.1. Let $\Sigma=\{a<b\}$ and $w \in \Sigma$. Let $w=a b a a b a b$,

$\delta_{2}(w)=\delta_{2}($ abaabab $)=\left(\begin{array}{lll}0 & 0 & 1 \\ 0 & 1 & 4 \\ 1 & 3 & 8\end{array}\right)$

$\delta_{2}\left(w^{t}\right)=\left(\begin{array}{lll}0 & 0 & 1 \\ 0 & 1 & 4 \\ 1 & 3 & 8\end{array}\right)^{t}=\left(\begin{array}{lll}0 & 0 & 1 \\ 0 & 1 & 3 \\ 1 & 4 & 8\end{array}\right)$

Since $(w)^{r e v}=(a b a a b a b)^{r e v}=b a b a a b a$, we get $\delta_{2}\left(w^{r e v}\right)=\delta_{2}(b a b a a b a)=$ $\left(\begin{array}{lll}0 & 0 & 1 \\ 0 & 1 & 4 \\ 1 & 3 & 4\end{array}\right)$

Therefore, $\delta_{2}\left(w^{t}\right) \neq \delta_{2}\left(w^{\text {rev }}\right)$

Theorem 4.1. Let $\Sigma=\{a, b\}$ with $a<b$. The word $\mathrm{s} w_{1}, w_{2}$ over $\Sigma$ satisfy transpose property, then $\left[\delta_{2}\left(w_{1} * w_{2}\right)\right]^{t}=\left[\delta_{2}\left(w_{2}\right)\right]^{t} *\left[\delta_{2}\left(w_{1}\right)\right]^{t}$. Here we mean $\delta_{2}\left(w_{1} * w_{2}\right)$ as $\delta_{2}\left(w_{1}\right) * \delta_{2}\left(w_{2}\right)$.

Proof: Let $\Sigma=\{a, b\}$ with $a<b$. Let $w_{1}, w_{2} \in \Sigma$.

$\delta_{2}\left(w_{1}\right)=\left(\begin{array}{lll}0 & 0 & 1 \\ 0 & 1 & p \\ 1 & q & r\end{array}\right)$ and 


$$
\begin{aligned}
& \delta_{2}\left(w_{2}\right)=\left(\begin{array}{ccc}
0 & 0 & 1 \\
0 & 1 & l \\
1 & m & n
\end{array}\right) \\
& \delta_{2}\left(w_{1} * w_{2}\right)=\delta_{2}\left(w_{1}\right) * \delta_{2}\left(w_{2}\right) \\
& =\left(\begin{array}{lll}
0 & 0 & 1 \\
0 & 1 & p \\
1 & q & r
\end{array}\right) *\left(\begin{array}{ccc}
0 & 0 & 1 \\
0 & 1 & l \\
1 & m & n
\end{array}\right) \\
& =\left(\begin{array}{ccc}
0 & 0 & 1 \\
0 & 1 & p+l \\
1 & q+m & r+p m+n
\end{array}\right) \text { as anti-diagonal matrix product } \\
& {\left[\delta_{2}\left(w_{1} * w_{2}\right)\right]^{t}=\left(\begin{array}{ccc}
0 & 0 & 1 \\
0 & 1 & p+l \\
1 & q+m & r+p m+n
\end{array}\right)^{t}=\left(\begin{array}{ccc}
0 & 0 & 1 \\
0 & 1 & q+m \\
1 & p+l & r+p m+n
\end{array}\right)} \\
& {\left[\delta_{2}\left(w_{1}\right)\right]^{t}=\left(\begin{array}{ccc}
0 & 0 & 1 \\
0 & 1 & p \\
1 & q & r
\end{array}\right)^{t}=\left(\begin{array}{ccc}
0 & 0 & 1 \\
0 & 1 & q \\
1 & p & r
\end{array}\right) \text { and }} \\
& {\left[\delta_{2}\left(w_{2}\right)\right]^{t}=\left(\begin{array}{ccc}
0 & 0 & 1 \\
0 & 1 & l \\
1 & m & n
\end{array}\right)^{t}=\left(\begin{array}{ccc}
0 & 0 & 1 \\
0 & 1 & m \\
1 & l & n
\end{array}\right)} \\
& {\left[\delta_{2}\left(w_{2}\right)\right]^{t} *\left[\delta_{2}\left(w_{1}\right)\right]^{t}=\left(\begin{array}{ccc}
0 & 0 & 1 \\
0 & 1 & m \\
1 & l & n
\end{array}\right) *\left(\begin{array}{ccc}
0 & 0 & 1 \\
0 & 1 & q \\
1 & p & r
\end{array}\right)} \\
& =\left(\begin{array}{ccc}
0 & 0 & 1 \\
0 & 1 & m+q \\
1 & l+p & n+m p+r
\end{array}\right) \text { as anti-diagonal matrix product. }
\end{aligned}
$$

Example 4.2. Let $w_{1}=a b a a b a b, \delta_{2}\left(w_{1}\right)=\left(\begin{array}{lll}0 & 0 & 1 \\ 0 & 1 & 4 \\ 1 & 3 & 8\end{array}\right)$ and $w_{2}=a b a b a$ and $\delta_{2}\left(w_{1}\right)=\left(\begin{array}{lll}0 & 0 & 1 \\ 0 & 1 & 3 \\ 1 & 2 & 3\end{array}\right)$

$\delta_{2}\left(w_{1} * w_{2}\right)=\delta_{2}\left(w_{1}\right) * \delta_{2}\left(w_{2}\right)=\left(\begin{array}{ccc}0 & 0 & 1 \\ 0 & 1 & 4 \\ 1 & 3 & 8\end{array}\right) *\left(\begin{array}{lll}0 & 0 & 1 \\ 0 & 1 & 3 \\ 1 & 2 & 3\end{array}\right)$

$=\left(\begin{array}{ccc}0 & 0 & 1 \\ 0 & 1 & 7 \\ 1 & 5 & 19\end{array}\right)$ as Anti-diagonal matrix product

$$
\begin{aligned}
& {\left[\delta_{2}\left(w_{1} * w_{2}\right)\right]^{t}=\left(\begin{array}{ccc}
0 & 0 & 1 \\
0 & 1 & 5 \\
1 & 7 & 19
\end{array}\right)} \\
& {\left[\delta_{2}\left(w_{1}\right)\right]^{t}=\left(\begin{array}{lll}
0 & 0 & 1 \\
0 & 1 & 4 \\
1 & 3 & 8
\end{array}\right)^{t}=\left(\begin{array}{lll}
0 & 0 & 1 \\
0 & 1 & 3 \\
1 & 4 & 8
\end{array}\right) \text { and }}
\end{aligned}
$$


Existence of Commutativity in Parikh and Anti-Diagonal Matrices

$$
\begin{aligned}
& {\left[\delta_{2}\left(w_{2}\right)\right]^{t}=\left(\begin{array}{lll}
0 & 0 & 1 \\
0 & 1 & 3 \\
1 & 2 & 3
\end{array}\right)^{t}=\left(\begin{array}{lll}
0 & 0 & 1 \\
0 & 1 & 2 \\
1 & 3 & 3
\end{array}\right) } \\
& {\left[\delta_{2}\left(w_{2}\right)\right]^{t} *\left[\delta_{2}\left(w_{1}\right)\right]^{t} }=\left(\begin{array}{lll}
0 & 0 & 1 \\
0 & 1 & 2 \\
1 & 3 & 3
\end{array}\right) *\left(\begin{array}{lll}
0 & 0 & 1 \\
0 & 1 & 3 \\
1 & 4 & 8
\end{array}\right) \\
&=\left(\begin{array}{ccc}
0 & 0 & 1 \\
0 & 1 & 5 \\
1 & 7 & 19
\end{array}\right)
\end{aligned}
$$

Hence, $\left[\delta_{2}\left(w_{1} * w_{2}\right)\right]^{t}=\left[\delta_{2}\left(w_{2}\right)\right]^{t} *\left[\delta_{2}\left(w_{1}\right)\right]^{t}$.

Theorem 4.2. Let $\Sigma=\{a, b\}$ with $a<b$. Let $w \in \Sigma$ satisfying transpose property, then $\left[\delta_{2}(w * m i(w))\right]^{t}=\left[\delta_{2}(m i(w))\right]^{t} *\left[\delta_{2}(w)\right]^{t}$.

Proof: Let $\Sigma=\{a, b\}$ with $a<b$. Let $w \in \Sigma$.

$\delta_{2}(w)=\left(\begin{array}{lll}0 & 0 & 1 \\ 0 & 1 & p \\ 1 & q & r\end{array}\right)$ and
$\delta_{2}(m i(w))=\left(\begin{array}{lll}0 & 0 & 1 \\ 0 & 1 & p \\ 1 & q & s\end{array}\right)$

The proof is similar to the proof of Theorem 4.1

\section{Conclusion}

The topic of Parikh matrices is a promising area of research related to combinatorics on words. In this paper we have obtained certain criteria for words so that their Parikh matrices and Anti-diagonal matrices are commute because of the non-commutativity of words. Most of problems are difficulties to handle mathematically in formal languages.

\section{REFERENCES}

1. A.Mateescu, A.Salomaa, K.Salomaa and S.Yu, On an extension of the Parikh mapping, Technical Report, 364, TUCS, 2000.

2. A.Mateescu, A.Salomaa, K.Salomaa and S.Yu, A sharpening of the Parikh mapping, Theoret. Informatics Appl., 35 (2001) 551-564.

3. A.Mateescu, A.Salomaa and S.Yu, Subword histories and Parikh matrices, J. Comput. Syst. Sci., 68 (2004) 1-21.

4. R.J.Parikh, On context-free languages, J. Assoc. Comput. Mach., 13 (1966) 570 581.

5. A.Salomaa, On the injectivity of the Parikh matrix mapping, Fundam. Informa., 64 (2005) 391- 404.

6. V.N.Serbanuta, Injectivity of the Parikh matrix mappings revisited, Fundamenta Informaticae, 73 (2006) 265-283.

7. T.F.Serbanuta, Extending Parikh matrices, Theoretical Computer Science, 310 (2004) 233-246.

8. R.StellaMaragatham and V.Nithya Vani, Anti-diagonal matrix: A variant of Parikh matrix, Proceedings of NCAGL2015. 
V. NithyaVani and R. Stella Maragatham

9. K.G.Subramanian, A.M.Huey, A.K.Nagar, On Parikh matrices, Int. J. Found. Comput. Sci., 20(2) (2009) $211-219$.

10. G.Rozenberg and A.Salomaa, (eds.), Handbook of Formal Languages 1-3. SpringerVerlag, Berlin, Heidelberg, New York (1997). 